\title{
THE DIMENSIONS OF INHOMOGENEOUS SELF-AFFINE SETS
}

\author{
Stuart A. Burrell and Jonathan M. Fraser \\ University of St Andrews, School of Mathematics and Statistics \\ St Andrews, KY16 9SS, UK; sb235@st-andrews.ac.uk \\ University of St Andrews, School of Mathematics and Statistics \\ St Andrews, KY16 9SS, UK; jmf32@st-andrews.ac.uk
}

\begin{abstract}
We prove that the upper box dimension of an inhomogeneous self-affine set is bounded above by the maximum of the affinity dimension and the dimension of the condensation set. In addition, we determine sufficient conditions for this upper bound to be attained, which, in part, constitutes an exploration of the capacity for the condensation set to mitigate dimension drop between the affinity dimension and the corresponding homogeneous attractor. Our work improves and unifies previous results on general inhomogeneous attractors, low-dimensional affine systems, and inhomogeneous self-affine carpets, while providing inhomogeneous analogues of Falconer's seminal results on homogeneous self-affine sets.
\end{abstract}

\section{Introduction}

A map $S: \mathbf{R}^{n} \rightarrow \mathbf{R}^{n}$ is affine if it can be written

$$
S(x)=A x+b
$$

for $A \in \mathrm{GL}(\mathbf{R}, n)$ and translation vector $b \in \mathbf{R}^{n}$, and is contracting if there exists $c \in(0,1)$ such that

$$
|S(x)-S(y)| \leq c|x-y|
$$

for all $x, y \in \mathbf{R}^{n}$. An affine iterated function system (IFS) is a finite collection $\left\{S_{i}\right\}_{i=1}^{N}$ of contracting affine maps. A classic application of Banach's contraction mapping theorem (for details, see [6]) proves the existence of a unique non-empty compact set $F$, called a homogeneous attractor, or self-affine set, such that

$$
F=\bigcup_{i=1}^{N} S_{i}(F) .
$$

There is a natural generalisation of this construction. If we fix a compact set $C \subseteq \mathbf{R}^{n}$, then there exists a unique non-empty compact set $F_{C}$ such that

$$
F_{C}=\bigcup_{i=1}^{N} S_{i}\left(F_{C}\right) \cup C,
$$

called the inhomogeneous attractor, or inhomogeneous self-affine set, with condensation set $C$. To express $F_{C}$ in an amenable way, we require some notation. Henceforth,

https://doi.org/10.5186/aasfm.2020.4516

2010 Mathematics Subject Classification: Primary 28A80.

Key words: Inhomogeneous attractor, self-affine set, box dimension, affinity dimension. 
let $\mathbf{I}=\left\{S_{i}\right\}_{i=1}^{N}$ denote an affine IFS and $\mathcal{I}=\{1, \ldots, N\}$. We write $S_{\mathbf{i}}=S_{i_{1}} \circ \cdots \circ S_{i_{k}}$ for $\mathbf{i}=\left(i_{1}, i_{2}, \ldots, i_{k}\right) \in \mathcal{I}^{k}$. Furthermore, let

$$
\mathcal{I}^{*}=\bigcup_{k=1}^{\infty} \mathcal{I}^{k}
$$

denote the set of finite words over $\mathcal{I}$. An elegant description of $F_{C}$, from [10] and [18], is

$$
F_{C}=F_{\emptyset} \cup \mathcal{O}
$$

where $F_{\emptyset}$ is the homogeneous attractor (corresponding to $C=\emptyset$ ), and $\mathcal{O}$ is the orbital set defined by

$$
\mathcal{O}=C \cup \bigcup_{\mathbf{i} \in \mathcal{I}^{*}} S_{\mathbf{i}}(C)
$$

Since their introduction by Barnsley (1985) [3] and Hata (1985) [13], inhomogeneous attractors have received further attention in, for example, [1, 4, 10, 11, 16, 17, 18]. A natural question explored in recent work concerns the relationship between the dimensions of $F_{C}, C$ and $F_{\emptyset}$. In particular, one may wonder in what situations

$$
\operatorname{dim} F_{C}=\max \left\{\operatorname{dim} F_{\emptyset}, \operatorname{dim} C\right\},
$$

where dim denotes some notion of dimension. For dimensions satisfying countable stability, such as the Hausdorff or packing dimension, this is immediate. Consequently, the recent focus has been on box dimension, a popular example of a countably unstable dimension. Recall that for a non-empty bounded set $F \subseteq \mathbf{R}^{n}$, the upper and lower box dimensions are defined as

$$
\overline{\operatorname{dim}}_{\mathrm{B}} F=\limsup _{\delta \rightarrow 0} \frac{\log N_{\delta}(F)}{-\log \delta},
$$

and

$$
\underline{\operatorname{dim}}_{\mathrm{B}} F=\liminf _{\delta \rightarrow 0} \frac{\log N_{\delta}(F)}{-\log \delta},
$$

respectively, where $N_{\delta}(F)$ denotes the minimum number of hypercubes of sidelength $\delta$ required to cover $F$. If these values coincide, we say the set has box dimension equal to the common value and denote this by $\operatorname{dim}_{\mathrm{B}} F$.

In $[1,10,17,18]$, various solutions to (1.1) are given for upper box dimension in the case where $\mathbf{I}$ consists of similarity mappings. For systems containing arbitrary bi-Lipschitz maps, bounds on $\overline{\operatorname{dim}}_{\mathrm{B}} F_{C}$ are given by Burrell based on upper Lipschitz dimension [4]. Corollaries of this result establish (1.1) for some low-dimensional affine systems and those satisfying bounded distortion, such as conformal systems (see [8] for definitions). For upper box dimension, (1.1) may fail for self-similar sets with overlaps [1] and specific self-affine settings [11]. In the case of lower box dimension, (1.1) fails to hold generally even for self-similar systems satisfying the strong separation condition [10].

The typical strategy used to approach (1.1), introduced in [10], is to establish bounds of the form

$$
\max \left\{\overline{\operatorname{dim}}_{\mathrm{B}} F_{\emptyset}, \overline{\operatorname{dim}}_{\mathrm{B}} C\right\} \leq \overline{\operatorname{dim}}_{\mathrm{B}} F_{C} \leq \max \left\{s, \overline{\operatorname{dim}}_{\mathrm{B}} C\right\},
$$

where $s \in \mathbf{R}$ is a natural estimate for $\overline{\operatorname{dim}}_{\mathrm{B}} F_{\emptyset}$, such as similarity dimension in the selfsimilar case [10] or upper Lipschitz dimension [4] in the general case. This exploits the abundance of literature on the equality of $s$ and $\overline{\operatorname{dim}}_{B} F_{\emptyset}$ in different settings, which may then determine precise conditions for equality. In the affine setting, the 
natural candidate for $s$ is the affinity dimension, and we prove (1.2) holds in this case.

The affinity dimension is derived from the notion of Falconer's singular value function, introduced in [5]. The singular values of $A \in \mathrm{GL}(\mathbf{R}, n)$ are written $\alpha_{j}(A)$ (or simply $\alpha_{j}$ ) and correspond to the lengths of the mutually perpendicular principal axes of $A(B)$, where $B$ denotes a ball of unit diameter in $\mathbf{R}^{n}$ [5]. Alternatively, they are the positive square roots of the eigenvalues of $A A^{T}$. We adopt the convention $1>\alpha_{1} \geq \alpha_{2} \geq \cdots \geq \alpha_{n}>0$. For $0 \leq s \leq n$, the singular value function of $A \in \mathrm{GL}(\mathbf{R}, n)$ is given by

$$
\phi^{s}(A)=\alpha_{1}(A) \alpha_{2}(A) \cdots \alpha_{m}(A)^{s-m+1},
$$

where $m \in \mathbf{Z}$ satisfies $m-1<s \leq m$. Finally, as in [5], we define $\phi^{s}(A)=(\operatorname{det} T)^{s / n}$ for $s>n$. Moreover, for convenience we set $\phi^{s}(S)=\phi^{s}(A)$ where $A$ is the linear component of a general affine map $S$. Then, for each $k \in \mathbf{N}$, define $s_{k}$ to be the solution of

$$
\sum_{\mathbf{i} \in \mathcal{I}^{k}} \phi^{s_{k}}\left(S_{\mathbf{i}}\right)=1
$$

The corresponding limit, denoted throughout by $s$,

$$
s:=\lim _{k \rightarrow \infty} s_{k},
$$

exists and is known as the affinity dimension associated with $\mathbf{I}$. It is proven in [4] that if the affinity dimension $s$ is less than or equal to 1 and coincides with $\overline{\operatorname{dim}}_{\mathrm{B}} F_{\emptyset}$, then

$$
\overline{\operatorname{dim}}_{\mathrm{B}} F_{C}=\max \left\{\overline{\operatorname{dim}}_{\mathrm{B}} F_{\emptyset}, \overline{\operatorname{dim}}_{\mathrm{B}} C\right\} .
$$

This is an immediate corollary of [4, Theorem 2.1], arising from the fact that when the affinity dimension is less than one it coincides with the upper Lipschitz dimension. Otherwise, it is elementary to see that the affinity dimension is generally strictly less than the upper Lipschitz dimension. Thus, establishing (1.2) for affinity dimension constitutes a natural and strictly improved bound for affine systems in comparison to the universal bound from [4].

\section{Results}

Our main result may be considered an inhomogeneous analogue of Falconer's seminal result on homogeneous self-affine sets [5], which establishes $\overline{\operatorname{dim}}_{\mathrm{B}} F_{\emptyset} \leq s$.

Theorem 2.1. Let $F_{C} \subset \mathbf{R}^{n}$ be an inhomogeneous self-affine set with compact condensation set $C \subset \mathbf{R}^{n}$. We have

$$
\max \left\{\overline{\operatorname{dim}}_{\mathrm{B}} F_{\emptyset}, \overline{\operatorname{dim}}_{\mathrm{B}} C\right\} \leq \overline{\operatorname{dim}}_{\mathrm{B}} F_{C} \leq \max \left\{s, \overline{\operatorname{dim}}_{\mathrm{B}} C\right\},
$$

where $s$ is the affinity dimension associated with the underlying IFS.

The following corollary is immediate.

Corollary 2.2. Let $F_{C} \subset \mathbf{R}^{n}$ be an inhomogeneous self-affine set with compact condensation set $C \subset \mathbf{R}^{n}$ and let $s$ be the associated affinity dimension. Then

(1) if $\overline{\operatorname{dim}}_{\mathrm{B}} F_{\emptyset}=s$, then $\overline{\operatorname{dim}}_{\mathrm{B}} F_{C}=\max \left\{\overline{\operatorname{dim}}_{\mathrm{B}} F_{\emptyset}, \overline{\operatorname{dim}}_{\mathrm{B}} C\right\}$,

(2) if $\overline{\operatorname{dim}}_{\mathrm{B}} C \geq s$, then $\overline{\operatorname{dim}}_{\mathrm{B}} F_{C}=\overline{\operatorname{dim}}_{\mathrm{B}} C$. 
Establishing precise conditions for the affinity dimension to coincide with $\overline{\operatorname{dim}}_{\mathrm{B}} F_{\emptyset}$ is a major open problem in fractal geometry and has been the focus of considerable amounts of work, for example $[2,5,7,9,11,12,14,15]$. Therefore there are numerous explicit and non-explicit situations where Corollary 2.2 provides a precise result, and an affirmative solution to (1.1) in the self-affine setting. For example, a wellknown result by Falconer [5] states that $s=\overline{\operatorname{dim}}_{\mathrm{B}} F_{\emptyset}=\operatorname{dim}_{\mathrm{H}} F_{\emptyset}$ almost surely if one randomises the translation vectors associated with the affine maps, provided the linear parts all have norm strictly bounded above by $1 / 2$, see also [15]. Falconer proved in a subsequent paper that if $F_{\emptyset} \subset \mathbf{R}^{2}$ satisfies some separation conditions and contains a connected component not contained in a straight line, then $s=\overline{\operatorname{dim}}_{\mathrm{B}} F_{\emptyset}$ holds, see [7, Corollary 5]. A recent breakthrough result of Bárány, Hochman and Rapaport [2] proves $s=\overline{\operatorname{dim}}_{\mathrm{B}} F_{\emptyset}=\operatorname{dim}_{\mathrm{H}} F_{\emptyset}$ in the planar case assuming only strong separation, together with mild non-compactness and irreducibility assumptions on the linear components of the maps $S_{i}$.

The next result explores the case where $\overline{\operatorname{dim}}_{\mathrm{B}} F_{C}>\max \left\{\overline{\operatorname{dim}}_{\mathrm{B}} F_{\emptyset}, \overline{\operatorname{dim}}_{\mathrm{B}} C\right\}$, that is when (1.1) fails. This is an exploration of conditions under which $C$ compensates for dimension drop between $s$ and $\overline{\operatorname{dim}}_{\mathrm{B}} F_{\emptyset}$. To state this result, we require the definition of the condensation open set condition (COSC), appearing in $[16,17,18]$ and $m$ - $\delta$-stoppings. Firstly, an IFS satisfies the COSC if there exists an open set $U$ with

$$
C \subset U \backslash \bigcup_{i=1}^{N} \overline{S_{i}(U)},
$$

such that $S_{i}(U) \subset U$ for $i=1, \ldots, N$, and $i \neq j \Longrightarrow S_{i}(U) \cap S_{j}(U)=\emptyset$. Secondly, for each $1 \leq m \leq n$ and $\delta \in(0,1]$, define the $m$ - $\delta$-stopping to be

$$
\mathcal{I}_{m}(\delta)=\left\{\mathbf{i} \in \mathcal{I}^{*}: \alpha_{m}\left(S_{\mathbf{i}}\right)<\delta \leq \alpha_{m}\left(S_{\mathbf{i}_{-}}\right)\right\}
$$

where $\mathbf{i}_{-}=\left(i_{1}, \ldots, i_{k-1}\right)$ for $\mathbf{i}=\left(i_{1}, \ldots, i_{k}\right)$. For the next theorem we will only use $\mathcal{I}_{n}(\delta)$, but later in the proofs section we will use it more generally and so introduced it here in full generality for brevity. Throughout, we fix a compact ball $X \subset \mathbf{R}^{n}$ such that $S_{i}(X) \subset X$ for $i=1, \ldots, N$ and $C \subseteq X$. Such a ball always exists and without loss of generality, we may assume that $X$ has unit diameter.

Theorem 2.3. Let $\mathbf{I}=\left\{S_{i}\right\}_{i=1}^{N}$ denote an affine IFS with condensation set $C \subseteq$ $\mathbf{R}^{n}$ satisfying the COSC. If $\underline{\operatorname{dim}}_{\mathrm{B}} C \geq n-1$ and there exists $\kappa>0$ such that for all $\delta \in(0,1]$ and $\boldsymbol{i} \in \mathcal{I}_{n}(\delta)$ we have

$$
N_{\delta}\left(S_{\mathbf{i}}(C)\right) \geq \kappa N_{\delta}\left(S_{\mathbf{i}}(X)\right),
$$

then

and

$$
\overline{\operatorname{dim}}_{\mathrm{B}} F_{C}=\max \left\{s, \overline{\operatorname{dim}}_{\mathrm{B}} C\right\}
$$

$$
\max \left\{s, \underline{\operatorname{dim}}_{\mathrm{B}} C\right\} \leq \underline{\operatorname{dim}}_{\mathrm{B}} F_{C} \leq \max \left\{s, \overline{\operatorname{dim}}_{\mathrm{B}} C\right\} .
$$

Note that the condition of the theorem is independent of the choice of ball $X$, although the constant $\kappa$ may change. The fact that we only get bounds for the lower box dimension of $F_{C}$ should not come as a surprise and one should not expect to be able to improve these bounds in general, see [10]. Note that if, in the setting of Theorem 2.3, the box dimension of $C$ exists, then so does the box dimension of $F_{C}$.

The assumption in Theorem 2.3 arises in quite natural circumstances, for example, the setting of the following proposition, an inhomogeneous analogue of Falconer's [7, Proposition 4], requires only that $C$ be in some sense robust under projection onto 
subspaces. Let $\mathcal{L}^{k}$ denote $k$-dimensional Lebesgue measure and $P_{k}$ denote the set of orthogonal projections onto $k$-dimensional subspaces of $\mathbf{R}^{n}$.

Proposition 2.4. Let $F_{C} \subset \mathbf{R}^{n}$ be an inhomogeneous self-affine set with compact condensation set $C \subset \mathbf{R}^{n}$ satisfying the COSC and let $s$ be the associated affinity dimension. If

$$
\inf _{\pi \in P_{n-1}} \mathcal{L}^{n-1}(\pi C)>0
$$

then

$$
\overline{\operatorname{dim}}_{\mathrm{B}} F_{C}=\max \left\{s, \overline{\operatorname{dim}}_{\mathrm{B}} C\right\}
$$

and

$$
\max \left\{s, \underline{\operatorname{dim}}_{\mathrm{B}} C\right\} \leq \underline{\operatorname{dim}}_{\mathrm{B}} F_{C} \leq \max \left\{s, \overline{\operatorname{dim}}_{\mathrm{B}} C\right\} .
$$

The robustness assumption on $C$ in Proposition 2.4 forces $\underline{\operatorname{dim}}_{\mathrm{B}} C \geq n-1$ and so this result only yields new information when $s>n-1$. It is interesting to compare this result with [4, Corollary 2.3] and the discussion thereafter, which applies to self-affine systems where $s \leq 1$.

The projection of a connected set in $\mathbf{R}^{2}$ which is not contained in a line onto a line contains an interval with length uniformly bounded away from 0 . This observation yields the following corollary of Proposition 2.4.

Corollary 2.5. Let $F_{C} \subset \mathbf{R}^{2}$ be an inhomogeneous self-affine set with compact condensation set $C \subset \mathbf{R}^{2}$ satisfying the COSC and let $s$ be the associated affinity dimension. If $C$ has a connected component not contained in a line, then

$$
\overline{\operatorname{dim}}_{\mathrm{B}} F_{C}=\max \left\{s, \overline{\operatorname{dim}}_{\mathrm{B}} C\right\}
$$

and

$$
\max \left\{s, \underline{\operatorname{dim}}_{\mathrm{B}} C\right\} \leq \underline{\operatorname{dim}}_{\mathrm{B}} F_{C} \leq \max \left\{s, \overline{\operatorname{dim}}_{\mathrm{B}} C\right\} .
$$

The reader may find it interesting to notice the parallels between this result and Falconer's [7, Corollary 5], which concerns the equality of $\overline{\operatorname{dim}}_{\mathrm{B}} F_{\emptyset}$ and $s$ under similar conditions concerning the robustness of connected components under projection. In some sense our inhomogeneous analogue is easier to use than the homogeneous result of Falconer. Our result requires a connectedness condition on $C$, which is given, whereas the homogeneous result requires one to check a connectedness condition on $F_{\emptyset}$, which depends delicately on the IFS. Moreover, the separation assumption makes it difficult for $F_{\emptyset}$ to be connected at all. For example, the strong separation condition forces $F_{\emptyset}$ to be totally disconnected, but our result can still apply in this setting.

The above results provide new families of inhomogeneous attractors where (1.1) fails for the upper (and lower) box dimension. We illustrate this by example. Let $n=2$ and $\mathbf{I}=\left\{S_{1}, S_{2}\right\}$, where $S_{1}, S_{2}$ are the linear maps associated with the matrices

$$
\left[\begin{array}{cc}
1 / 2 & 0 \\
1 / 2 & 1 / 2
\end{array}\right], \quad\left[\begin{array}{cc}
1 / 2 & 1 / 2 \\
0 & 1 / 2
\end{array}\right]
$$

respectively. It is clear that the affinity dimension of this system is strictly greater than one and that $F_{\emptyset}$ is just a single point at the origin. Let $C$ be the boundary of a circle centred at $(3 / 4,3 / 4)$ with radius $1 / 5$. It is also clear that the COSC is satisfied by taking $U=(0,1)^{2}$ and that $C$ is connected but not contained in a line, see Figure 1. It follows from Corollary 2.5 that

$$
\overline{\operatorname{dim}}_{\mathrm{B}} F_{C}=\underline{\operatorname{dim}}_{\mathrm{B}} F_{C}=s>1=\max \left\{\operatorname{dim}_{\mathrm{B}} F_{\emptyset}, \operatorname{dim}_{\mathrm{B}} C\right\} .
$$



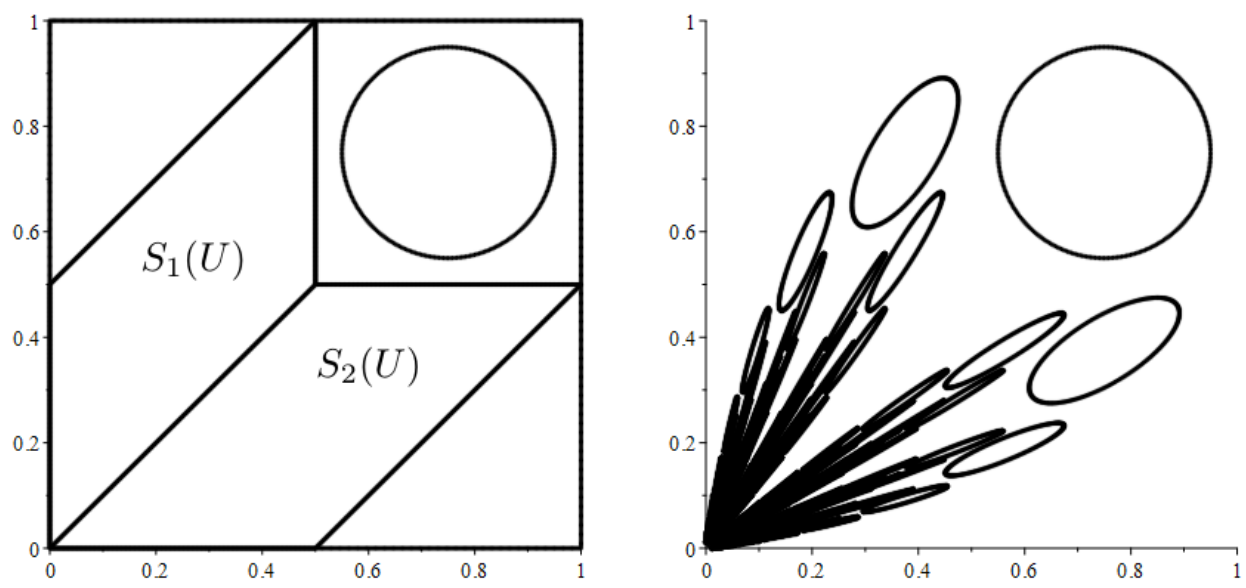

Figure 1. A bouquet of ovals: the condensation set together with the two images of the open rectangle $U=(0,1)^{2}$ (left) and the corresponding inhomogeneous self-affine set (right).

This is the first counter example to (1.1) where $F_{\emptyset}$ is a single point and the OSC is satisfied. Moreover, it was shown in [1, Corollary 4.9] that for planar inhomogeneous self-similar sets one always has

$$
\overline{\operatorname{dim}}_{\mathrm{B}} F_{C} \leq \max \left\{\overline{\operatorname{dim}}_{\mathrm{B}} C, \overline{\operatorname{dim}}_{\mathrm{B}} F_{\emptyset}+\overline{\operatorname{dim}}_{\mathrm{B}} C-\frac{\overline{\operatorname{dim}}_{\mathrm{B}} F_{\emptyset} \overline{\operatorname{dim}}_{\mathrm{B}} C}{s}\right\}
$$

where $s$ is the similarity dimension. In particular this shows that when $\overline{\operatorname{dim}}_{\mathrm{B}} F_{\emptyset}=0$ the formula (1.1) cannot fail. The example presented above shows that this phenomenon does not extend to the self-affine case. It was also shown in [1, Corollary 4.8] that, in the self-similar setting, if $\max \left\{\overline{\operatorname{dim}}_{\mathrm{B}} F_{\emptyset}, \overline{\operatorname{dim}}_{\mathrm{B}} C\right\}<s$, then $\overline{\operatorname{dim}}_{\mathrm{B}} F_{C}<s$. The above example also demonstrates that this does not extend to the self-affine setting.

The assumption in Proposition 2.4 is by no means necessary, and advancements in the homogeneous setting may illuminate further the capacity for $C$ to mitigate dimension drop. Excitingly, we suggest the natural interplay between these questions may allow further study of inhomogeneous attractors to translate into novel conditions relating to dimension drop in the homogeneous case. Specifically, this may arise from solutions to the following.

Question 1. Consider an affine IFS $\mathbf{I}=\left\{S_{i}\right\}_{i=1}^{N}$ with condensation set $C \subseteq \mathbf{R}^{n}$. If $s>\overline{\operatorname{dim}}_{\mathrm{B}} F_{\emptyset}$, then what conditions guarantee

$$
\overline{\operatorname{dim}}_{\mathrm{B}} F_{C}=\max \left\{s, \overline{\operatorname{dim}}_{\mathrm{B}} C\right\} ?
$$

\section{Proof of Theorem 2.1}

Let $\mathbf{I}=\left\{S_{i}\right\}_{i=1}^{N}$ be an affine IFS and $C \subseteq X$ be compact. Denote the affinity dimension of $\mathbf{I}$ by $s$ and assume $s \leq n$, since if $s>n$ the result is trivial.

It follows immediately from the definition of box dimension that for $t>\overline{\operatorname{dim}}_{\mathrm{B}} C$ there exists a constant $A_{t}$ satisfying

$$
N_{\delta}(C) \leq A_{t} \delta^{-t}
$$

for all $\delta \in(0,1]$. In addition, if $t>s$, then

$$
B_{t}:=\sum_{\mathbf{i} \in \mathcal{I}^{*}} \phi^{t}\left(S_{\mathbf{i}}\right)<\infty
$$


by $\left[5\right.$, Proposition 4.1 (c)], where $B_{t}$ depends only on $t$. We fix a constant $b \in \mathbf{R}$ satisfying

$$
0<b<\min _{i=1, \ldots, N} \alpha_{n}\left(S_{i}\right)<1,
$$

and note for any $\delta \in(0,1], 1 \leq m \leq n$ and $\mathbf{i} \in \mathcal{I}_{m}(\delta)$, we have

$$
\delta \geq \alpha_{m}\left(S_{\mathbf{i}}\right) \geq \alpha_{m}\left(S_{\mathbf{i}_{-}}\right) b \geq \delta b .
$$

Prior to reading the subsequent arguments, the following simple geometric observation, employed frequently in our proofs, may aid the reader less familiar with the classical arguments on self-affine sets found in [5] or [6]. Consider an ellipsoid $E$ with principal axes of lengths $l_{1}, \ldots, l_{n}$. For dimension calculations, we are interested in obtaining an estimate of the number of hypercubes of a given sidelength required to cover such ellipsoids. Constants are typically inconsequential, and so often a coarse estimate suffices. The minimum number of hypercubes of sidelength $l_{m}$ required to cover $E$ is at most

$$
\left(\frac{l_{1}}{l_{m}}+1\right)\left(\frac{l_{2}}{l_{m}}+1\right) \cdots\left(\frac{l_{m-1}}{l_{m}}+1\right) \leq 2^{n} \frac{l_{1}}{l_{m}} \frac{l_{2}}{l_{m}} \cdots \frac{l_{m-1}}{l_{m}}=2^{n} l_{1} l_{2} \cdots l_{m-1} l_{m}^{-m+1}
$$

This can be seen by first covering $E$ by a minimal hypercuboid of sidelengths equal to the principal axes of $E$ and then covering this optimally. Figure 2 illustrates this fact for a cuboid of sidelengths $a>b>c$ in $\mathbf{R}^{3}$. Specifically, we see that $2 a / b$ cubes of sidelength $b$ would suffice, whereas we would require a single cube of sidelength $a$ or at most $2^{2}(a / c)(b / c)$ cubes of sidelength $c$.

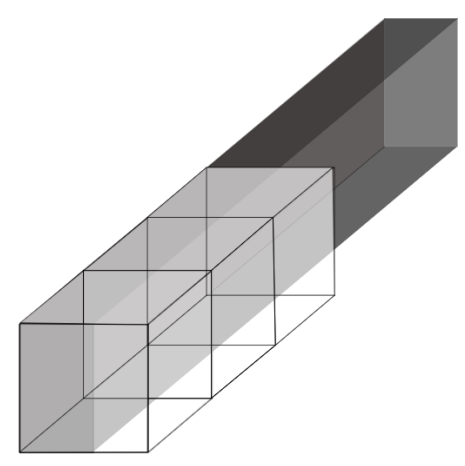

Figure 2. Covering a cuboid of sidelengths $a>b>c$ in $\mathbf{R}^{3}$ with cubes of sidelength $b$.

\subsection{Preliminary lemmas.}

Lemma 3.1. For $\delta \in(0,1]$ and $1 \leq m \leq n$, we have

Proof. For

$$
\bigcup_{\substack{i \in \mathcal{I}^{*} \\ \delta>\alpha_{m}\left(S_{\mathbf{i}}\right)}} S_{\mathbf{i}}(C) \subseteq \bigcup_{\mathbf{i} \in \mathcal{I}_{m}(\delta)} S_{\mathbf{i}}(X)
$$

$$
x \in \bigcup_{\substack{\mathbf{i} \in \mathcal{I}^{*} \\ \delta>\alpha_{m}\left(S_{\mathbf{i}}\right)}} S_{\mathbf{i}}(C),
$$

there exists some $\mathbf{i}=\left(i_{1}, \ldots, i_{k}\right) \in \mathcal{I}^{*}$ such that $x \in S_{\mathbf{i}}(C)$ and $\delta>\alpha_{m}\left(S_{\mathbf{i}}\right)$. Since $\delta>\alpha_{m}\left(S_{\mathbf{i}}\right)$, there also exists some prefix $\mathbf{i}_{p}$ of $\mathbf{i}$ with $\mathbf{i}_{p} \in \mathcal{I}_{m}(\delta)$, and so let us consider 
the concatenation $\mathbf{i}=\mathbf{i}_{p} \mathbf{j}$. If $\mathbf{j}=\emptyset$, then $\mathbf{i} \in \mathcal{I}_{m}(\delta)$. Else, there exists some $c$ such that $x=S_{\mathbf{i}}(c)=S_{\mathbf{i}_{p}}\left(S_{\mathbf{j}}(c)\right) \in S_{\mathbf{i}_{p}}(X)$ as required.

Lemma 3.2. Fix $1 \leq m \leq n$ and let $\boldsymbol{i} \in \mathcal{I}^{*}$ be such that $\alpha_{m}\left(S_{\boldsymbol{i}}\right)<\delta$. We have

$$
N_{\delta}\left(S_{\mathbf{i}}(X)\right) \leq 2^{n} \frac{\alpha_{1}\left(S_{\mathbf{i}}\right)}{\alpha_{m}\left(S_{\mathbf{i}}\right)} \frac{\alpha_{2}\left(S_{\mathbf{i}}\right)}{\alpha_{m}\left(S_{\mathbf{i}}\right)} \cdots \frac{\alpha_{m-1}\left(S_{\boldsymbol{i}}\right)}{\alpha_{m}\left(S_{\boldsymbol{i}}\right)} .
$$

Proof. First note that $S_{\mathbf{i}}(X)$ is an ellipsoid with principal axes having lengths equal to the singular values of $S_{\mathbf{i}}$. The result then follows follows immediately from the geometric observation described by equation (3.4).

Lemma 3.3. Fix $t \in(0, n]$ and let $m \in \mathbf{Z}$ be such that $m-1<t \leq m$. If $\boldsymbol{i} \in \mathcal{I}^{*}$ is such that $\alpha_{m}\left(S_{\mathbf{i}}\right) \geq \delta$, we have

$$
N_{\delta}\left(S_{\mathbf{i}}(C)\right) \leq 2^{n} A_{t} \delta^{-t} \phi^{t}\left(S_{\mathbf{i}}\right) .
$$

Proof. The image under $S_{\mathbf{i}}$ of a cover of $C$ by balls of diameter $\delta / \alpha_{m}\left(S_{\mathbf{i}}\right)$ is a cover of $S_{\mathbf{i}}(C)$ by ellipsoids with the $m$ largest principal axes of lengths

$$
\alpha_{i}\left(S_{\mathbf{i}}\right)\left(\frac{\delta}{\alpha_{m}\left(S_{\mathbf{i}}\right)}\right)=\delta \frac{\alpha_{i}\left(S_{\mathbf{i}}\right)}{\alpha_{m}\left(S_{\mathbf{i}}\right)}
$$

for $i=1, \ldots, m$, the smallest of which has length $\delta$. Each such ellipsoid can be covered by at most

$$
\frac{2 \delta \frac{\alpha_{1}\left(S_{\mathbf{i}}\right)}{\alpha_{m}\left(S_{\mathbf{i}}\right)}}{\delta} \frac{2 \delta \frac{\alpha_{2}\left(S_{\mathbf{i}}\right)}{\alpha_{m}\left(S_{\mathbf{i}}\right)}}{\delta} \cdots \frac{2 \delta \frac{\alpha_{m-1}\left(S_{\mathbf{i}}\right)}{\alpha_{m}\left(S_{\mathbf{i}}\right)}}{\delta} \leq 2^{n} \frac{\alpha_{1}\left(S_{\mathbf{i}}\right)}{\alpha_{m}\left(S_{\mathbf{i}}\right)} \frac{\alpha_{2}\left(S_{\mathbf{i}}\right)}{\alpha_{m}\left(S_{\mathbf{i}}\right)} \cdots \frac{\alpha_{m-1}\left(S_{\mathbf{i}}\right)}{\alpha_{m}\left(S_{\mathbf{i}}\right)}
$$

hypercubes of sidelength $\delta$. Hence

$$
\begin{aligned}
N_{\delta}\left(S_{\mathbf{i}}(C)\right) & \leq N_{\delta / \alpha_{m}\left(S_{\mathbf{i}}\right)}(C)\left(2^{n} \frac{\alpha_{1}\left(S_{\mathbf{i}}\right)}{\alpha_{m}\left(S_{\mathbf{i}}\right)} \frac{\alpha_{2}\left(S_{\mathbf{i}}\right)}{\alpha_{m}\left(S_{\mathbf{i}}\right)} \cdots \frac{\alpha_{m-1}\left(S_{\mathbf{i}}\right)}{\alpha_{m}\left(S_{\mathbf{i}}\right)}\right) \\
& \leq A_{t}\left(\frac{\delta}{\alpha_{m}\left(S_{\mathbf{i}}\right)}\right)^{-t}\left(2^{n} \frac{\alpha_{1}\left(S_{\mathbf{i}}\right)}{\alpha_{m}\left(S_{\mathbf{i}}\right)} \frac{\alpha_{2}\left(S_{\mathbf{i}}\right)}{\alpha_{m}\left(S_{\mathbf{i}}\right)} \cdots \frac{\alpha_{m-1}\left(S_{\mathbf{i}}\right)}{\alpha_{m}\left(S_{\mathbf{i}}\right)}\right) \\
& =2^{n} A_{t} \delta^{-t} \phi^{t}\left(S_{\mathbf{i}}\right)
\end{aligned}
$$

as required.

3.2. Proof of Theorem 2.1. Monotonicity and finite stability of upper box dimension imply

$$
\max \left\{\overline{\operatorname{dim}}_{\mathrm{B}} F_{\emptyset}, \overline{\operatorname{dim}}_{\mathrm{B}} C\right\} \leq \overline{\operatorname{dim}}_{\mathrm{B}} F_{C} \leq \max \left\{\overline{\operatorname{dim}}_{\mathrm{B}} F_{\emptyset}, \overline{\operatorname{dim}}_{\mathrm{B}} \mathcal{O}\right\}
$$

and so it suffices to show that

$$
\overline{\operatorname{dim}}_{\mathrm{B}} \mathcal{O} \leq \max \left\{s, \overline{\operatorname{dim}}_{\mathrm{B}} C\right\}
$$

since it is well known (see [6, Theorem 9.12]) that $s \geq \overline{\operatorname{dim}}_{\mathrm{B}} F_{\emptyset}$. Fix $\delta \in(0,1]$ and $t>\max \left\{s, \overline{\operatorname{dim}}_{\mathrm{B}} C\right\}$. If $\max \left\{s, \overline{\operatorname{dim}}_{\mathrm{B}} C\right\} \geq n$ then the result is trivial, so we may 
assume $t \leq n$. For $m \in \mathbf{Z}$ satisfying $m-1<t \leq m$, we have

$$
\begin{aligned}
& \delta^{t} N_{\delta}(\mathcal{O})=\delta^{t} N_{\delta}\left(C \cup \bigcup_{\mathbf{i} \in \mathcal{I}^{*}} S_{\mathbf{i}}(C)\right) \\
& \leq A_{t}+\delta^{t} N_{\delta}\left(\bigcup_{\substack{\mathbf{i} \in \mathcal{I}^{*} \\
\alpha_{m}\left(S_{\mathbf{i}}\right) \geq \delta}} S_{\mathbf{i}}(C)\right)+\delta^{t} N_{\delta}\left(\bigcup_{\substack{\mathbf{i} \in \mathcal{I}^{*} \\
\alpha_{m}\left(S_{\mathbf{i}}\right)<\delta}} S_{\mathbf{i}}(C)\right) \quad(\operatorname{using}(3.1)) \\
& \leq A_{t}+\delta^{t} \sum_{\substack{\mathbf{i} \in \mathcal{I}^{*} \\
\alpha_{m}\left(S_{\mathbf{i}}\right) \geq \delta}} N_{\delta}\left(S_{\mathbf{i}}(C)\right)+\delta^{t} \sum_{\mathbf{i} \in \mathcal{I}_{m}(\delta)} N_{\delta}\left(S_{\mathbf{i}}(X)\right) \quad \text { (by Lemma 3.1) } \\
& \leq A_{t}+\delta^{t} \sum_{\substack{\mathbf{i} \in \mathcal{I}^{*} \\
\alpha_{m}\left(S_{\mathbf{i}}\right) \geq \delta}} 2^{n} A_{t} \delta^{-t} \phi^{t}\left(S_{\mathbf{i}}\right) \\
& +\delta^{t} \sum_{\mathbf{i} \in \mathcal{I}_{m}(\delta)} 2^{n} \frac{\alpha_{1}\left(S_{\mathbf{i}}\right)}{\alpha_{m}\left(S_{\mathbf{i}}\right)} \frac{\alpha_{2}\left(S_{\mathbf{i}}\right)}{\alpha_{m}\left(S_{\mathbf{i}}\right)} \cdots \frac{\alpha_{m-1}\left(S_{\mathbf{i}}\right)}{\alpha_{m}\left(S_{\mathbf{i}}\right)} \quad \text { (by Lemmas } 3.2 \text { and 3.3) } \\
& \leq A_{t}+2^{n} A_{t} \sum_{\substack{\mathbf{i} \in \mathcal{I}^{*} \\
\alpha_{m}\left(S_{\mathbf{i}}\right) \geq \delta}} \phi^{t}\left(S_{\mathbf{i}}\right) \\
& +2^{n} \sum_{\mathbf{i} \in \mathcal{I}_{m}(\delta)} \frac{\alpha_{1}\left(S_{\mathbf{i}}\right)}{\alpha_{m}\left(S_{\mathbf{i}}\right)} \frac{\alpha_{2}\left(S_{\mathbf{i}}\right)}{\alpha_{m}\left(S_{\mathbf{i}}\right)} \cdots \frac{\alpha_{m-1}\left(S_{\mathbf{i}}\right)}{\alpha_{m}\left(S_{\mathbf{i}}\right)} \frac{\alpha_{m}\left(S_{\mathbf{i}}\right)^{t}}{b^{t}} \quad(\operatorname{using}(3.3)) \\
& \leq A_{t}+2^{n} A_{t} \sum_{\substack{\mathbf{i} \in \mathcal{I}^{*} \\
\alpha_{m}\left(S_{\mathbf{i}}\right) \geq \delta}} \phi^{t}\left(S_{\mathbf{i}}\right)+\frac{2^{n}}{b^{t}} \sum_{\mathbf{i} \in \mathcal{I}_{m}(\delta)} \phi^{t}\left(S_{\mathbf{i}}\right) \\
& \leq A_{t}+2^{n} B_{t}\left(A_{t}+b^{-t}\right) \quad(\operatorname{using}(3.2))
\end{aligned}
$$

Thus,

$$
\frac{\log N_{\delta}(\mathcal{O})}{-\log \delta} \leq t+\frac{\log \left(A_{t}+2^{n} B_{t}\left(A_{t}+b^{-t}\right)\right)}{-\log \delta},
$$

from which the result follows as $\delta \rightarrow 0$.

\section{Proof of Theorem 2.3}

Fix $\delta \in(0,1)$ and recall that $s$ denotes the affinity dimension of $\mathbf{I}$. It is stated in [7] that for $t<s$ there exists $c_{t}>0$ with

$$
\sum_{\mathcal{I}_{n}(\delta)} \phi^{t}\left(S_{\mathbf{i}}\right) \geq c_{t}
$$

for some constant $c_{t}$ that does not depend on $\delta$. This follows immediately from $[5$, Proposition 4.1 (a)]. Since we assume $\operatorname{dim}_{\mathrm{B}} C \geq n-1$, if $s \leq n-1$, then Theorem 2.1 implies that $\overline{\operatorname{dim}}_{\mathrm{B}} F_{C}=\overline{\operatorname{dim}}_{\mathrm{B}} C=\max \left\{s, \overline{\operatorname{dim}}_{\mathrm{B}} C\right\}$, and also $\underline{\operatorname{dim}}_{\mathrm{B}} F_{C} \geq \underline{\operatorname{dim}}_{\mathrm{B}} C=$ $\max \left\{s, \underline{\operatorname{dim}}_{\mathrm{B}} C\right\}$. If $s>n$, then the result is trivial. Thus, henceforth we assume that $n-1<t<s \leq n$.

Let $U$ denote the open set satisfying the COSC. Compactness of $C$ implies that there exists some constant $\eta>0$ with

$$
\inf \left\{|x-y|: x \in C, y \in \bigcup_{i=1}^{N} S_{\mathbf{i}}(U) \cup\left(\mathbf{R}^{n} \backslash U\right)\right\}=2 \eta .
$$


Let $B(C, \eta)$ denote a closed $\eta$-neighborhood of $C$ and $E$ be a hypercube of sidelength $\delta$ in a minimal $\delta$-cover of $\mathcal{O}$. For $\mathbf{i} \in \mathcal{I}_{n}(\delta)$, we have $S_{\mathbf{i}}(B(C, \eta))$ is a neighborhood of $S_{\mathbf{i}}(C)$ satisfying

$$
S_{\mathbf{i}}(B(C, \eta)) \cap F_{C}=S_{\mathbf{i}}(C)
$$

and

$$
\inf \left\{|x-y|: x \in S_{\mathbf{i}}(C), y \notin S_{\mathbf{i}}(B(C, \eta))\right\} \geq \alpha_{n}\left(S_{\mathbf{i}}\right) \eta>b \delta \eta
$$

implying

$$
\inf \left\{|x-y|: x \in S_{\mathbf{i}}(C), y \in S_{\mathbf{j}}(C) \text { such that } \mathbf{i}, \mathbf{j} \in \mathcal{I}_{n}(\delta), \mathbf{i} \neq \mathbf{j}\right\}>2 b \delta \eta .
$$

Let $V_{n}$ denote the constant such that the area of an $n$-sphere of radius $2 b \eta \delta$ is $V_{n} \delta^{n}$. For the sets in $\left\{S_{\mathbf{i}}(C): \mathbf{i} \in \mathcal{I}_{n}(\delta)\right\}$ that intersect $E$ we can associate pairwise disjoint open sets in $E$ of volume at least $V_{n} \delta^{n} / 2^{n}$ (with this lower bound obtained at the vertices) and it therefore follows by a simple volume argument that $E$ can cover at most

$$
\frac{\delta^{n}}{\frac{1}{2^{n}} V_{n} \delta^{n}}=\left(2^{-n} V_{n}\right)^{-1}
$$

of the sets $\left\{S_{\mathbf{i}}(C): \mathbf{i} \in \mathcal{I}_{n}(\delta)\right\}$. Hence

$$
N_{\delta}(\mathcal{O}) \geq 2^{-n} V_{n} \sum_{\mathbf{i} \in \mathcal{I}_{n}(\delta)} N_{\delta}\left(S_{\mathbf{i}}(C)\right) .
$$

Our assumption on $C$ implies that for $\mathbf{i} \in \mathcal{I}_{n}(\delta)$ we have

$$
\begin{aligned}
N_{\delta}\left(S_{\mathbf{i}}(C)\right) & \geq \kappa N_{\delta}\left(S_{\mathbf{i}}(X)\right) \\
& \geq \kappa b^{n} N_{b \delta}\left(S_{\mathbf{i}}(X)\right) \\
& \geq \kappa b^{n} N_{\alpha_{n}\left(S_{\mathbf{i}}\right)}\left(S_{\mathbf{i}}(X)\right) \\
& \geq \kappa b^{n} c \frac{\alpha_{1}\left(S_{\mathbf{i}}\right)}{\alpha_{n}\left(S_{\mathbf{i}}\right)} \frac{\alpha_{2}\left(S_{\mathbf{i}}\right)}{\alpha_{n}\left(S_{\mathbf{i}}\right)} \cdots \frac{\alpha_{n-1}\left(S_{\mathbf{i}}\right)}{\alpha_{n}\left(S_{\mathbf{i}}\right)}
\end{aligned}
$$

for some constant $c>0$ only depending on $n$. This yields

$$
\begin{aligned}
N_{\delta}(\mathcal{O}) & \geq 2^{-n} V_{n} \sum_{\mathbf{i} \in \mathcal{I}_{n}(\delta)} N_{\delta}\left(S_{\mathbf{i}}(C)\right) \quad(\operatorname{using}(4.2)) \\
& \geq 2^{-n} V_{n} \sum_{\mathbf{i} \in \mathcal{I}_{n}(\delta)} \kappa b^{n} c \frac{\alpha_{1}\left(S_{\mathbf{i}}\right)}{\alpha_{n}\left(S_{\mathbf{i}}\right)} \frac{\alpha_{2}\left(S_{\mathbf{i}}\right)}{\alpha_{n}\left(S_{\mathbf{i}}\right)} \cdots \frac{\alpha_{n-1}\left(S_{\mathbf{i}}\right)}{\alpha_{n}\left(S_{\mathbf{i}}\right)} \quad \text { using (4.3)) } \\
& =\kappa b^{n} c 2^{-n} V_{n} \sum_{\mathbf{i} \in \mathcal{I}_{n}(\delta)} \phi^{t}\left(S_{\mathbf{i}}\right) \alpha_{n}\left(S_{\mathbf{i}}\right)^{-t} \\
& \geq \kappa b^{n} c 2^{-n} V_{n} \delta^{-t} \sum_{\mathbf{i} \in \mathcal{I}_{n}(\delta)} \phi^{t}\left(S_{\mathbf{i}}\right) \\
& \geq \kappa b^{n} c 2^{-n} V_{n} c_{t} \delta^{-t}(\text { by }(4.1)) .
\end{aligned}
$$

Hence $\underline{\operatorname{dim}}_{\mathrm{B}} \mathcal{O} \geq t$, from which it follows that $\overline{\operatorname{dim}}_{\mathrm{B}} F_{C} \geq \underline{\operatorname{dim}}_{\mathrm{B}} F_{C} \geq \underline{\operatorname{dim}}_{\mathrm{B}} \mathcal{O} \geq s$, proving the theorem. 


\section{Proof of Proposition 2.4}

Let $\mathbf{I}=\left\{S_{i}\right\}_{i=1}^{N}$ denote an affine IFS with compact condensation set $C \subseteq \mathbf{R}^{n}$ satisfying the COSC. Moreover, suppose

$$
\inf _{\pi \in P_{n-1}} \mathcal{L}^{n-1}(\pi C)>0 .
$$

By Theorem 2.3 it suffices to show that there exists $\kappa>0$ such that for all $\delta>0$ and $\mathbf{i} \in \mathcal{I}_{n}(\delta)$ we have

$$
N_{\delta}\left(S_{\mathbf{i}}(C)\right) \geq \kappa N_{\delta}\left(S_{\mathbf{i}}(X)\right) .
$$

Therefore, in order to reach a contradiction, assume that for arbitrarily small $\kappa>0$ we can find $\delta>0$ and $\mathbf{i} \in \mathcal{I}_{n}(\delta)$ such that

$$
N_{\delta}\left(S_{\mathbf{i}}(C)\right)<\kappa N_{\delta}\left(S_{\mathbf{i}}(X)\right) \leq \kappa 2^{n} \frac{\alpha_{1}\left(S_{\mathbf{i}}\right)}{\alpha_{n}\left(S_{\mathbf{i}}\right)} \frac{\alpha_{2}\left(S_{\mathbf{i}}\right)}{\alpha_{n}\left(S_{\mathbf{i}}\right)} \cdots \frac{\alpha_{n-1}\left(S_{\mathbf{i}}\right)}{\alpha_{n}\left(S_{\mathbf{i}}\right)},
$$

where the final inequality comes from Lemma 3.2. Let $\left\{E_{j}\right\}_{j}$ be an optimal cover of $S_{\mathbf{i}}(C)$ by hypercubes of sidelength $\delta$ and place each $E_{j}$ inside a ball $B_{j}$ of diameter $\sqrt{n} \delta$ and consider $\left\{S_{\mathbf{i}}^{-1} B_{j}\right\}_{j}$ which is a cover of $C$ by ellipsoids with axes of length $\sqrt{n} \delta / \alpha_{1}\left(S_{\mathbf{i}}\right), \ldots, \sqrt{n} \delta / \alpha_{n}\left(S_{\mathbf{i}}\right)$. Note that the longest axes of each of these ellipsoids are all parallel (by the singular value decomposition theorem, for example) and let $\pi$ denote projection onto the $(n-1)$-dimensional hyperplane orthogonal to the common direction of the longest axes of the ellipsoids $\left\{S_{\mathbf{i}}^{-1} B_{j}\right\}_{j}$. It follows that $\left\{\pi S_{\mathbf{i}}^{-1} B_{j}\right\}_{j}$ is a cover of $\pi(C)$ by sets, each of which is easily seen to have $(n-1)$-volume at most

$$
n^{(n-1) / 2} \frac{\delta}{\alpha_{1}\left(S_{\mathbf{i}}\right)} \frac{\delta}{\alpha_{2}\left(S_{\mathbf{i}}\right)} \cdots \frac{\delta}{\alpha_{n-1}\left(S_{\mathbf{i}}\right)}
$$

and therefore we can bound the $(n-1)$-volume of $\pi(C)$ above by

$$
\begin{aligned}
& \kappa 2^{n} \frac{\alpha_{1}\left(S_{\mathbf{i}}\right)}{\alpha_{n}\left(S_{\mathbf{i}}\right)} \frac{\alpha_{2}\left(S_{\mathbf{i}}\right)}{\alpha_{n}\left(S_{\mathbf{i}}\right)} \cdots \frac{\alpha_{n-1}\left(S_{\mathbf{i}}\right)}{\alpha_{n}\left(S_{\mathbf{i}}\right)} \times n^{(n-1) / 2} \frac{\delta}{\alpha_{1}\left(S_{\mathbf{i}}\right)} \frac{\delta}{\alpha_{2}\left(S_{\mathbf{i}}\right)} \cdots \frac{\delta}{\alpha_{n-1}\left(S_{\mathbf{i}}\right)} \\
& \leq \kappa 2^{n} n^{(n-1) / 2} b^{-(n-1)}
\end{aligned}
$$

using (3.3). This contradicts the assumption that $\inf _{\pi \in P_{n-1}} \mathcal{L}^{n-1}(\pi C)>0$ since we can choose $\kappa$ arbitrarily small.

Acknowledgments. SAB thanks the Carnegie Trust for financially supporting this work. JMF was financially supported by a Leverhulme Trust Research Fellowship (RF-2016-500) and an EPSRC Standard Grant (EP/R015104/1).

\section{References}

[1] BAKer, S., J. M. FrAser, and Á. MÁthÉ: Inhomogeneous self-similar sets with overlaps. Ergodic Theory Dynam. Systems 39:1, 2019, 1-18.

[2] BÁrÁny, B., M. Hochman, and A. Rapaport: Hausdorff dimension of planar self affine sets and measures. - Invent. Math. (to appear).

[3] Barnsley, M. F., and S. Demko: Iterated function systems and the global construction of fractals. - Proc. R. Soc. Lond. Ser. A 399, 1985, 243-275.

[4] Burrell, S. A.: On the dimension and measure of inhomogeneous attractors. - Real Anal. Exchange 44:1, 2019, 199-216.

[5] Falconer, K. J.: The Hausdorff dimension of self-affine fractals. - Math. Proc. Camb. Phil. Soc. 103, 1988, 339-350.

[6] Falconer, K. J.: Fractal geometry: Mathematical foundations and applications. - John Wiley \& Sons, 1990. 
[7] Falconer, K. J.: The Hausdorff dimension of self-affine fractals II. - Math. Proc. Camb. Phil. Soc. 111, 1992, 169-179.

[8] Feng, D., and H. Hu: Dimension theory of iterated function systems. - Comm. Pure Appl. Math. 62:11, 2009, 1435-1500.

[9] Fraser, J. M.: On the packing dimension of box-like self-affine sets in the plane. - Nonlinearity $15: 1,2012,77-97$.

[10] Fraser, J. M.: Inhomogeneous self-similar sets and box dimensions. - Studia Math. 213, 2012, 133-156.

[11] Fraser, J. M.: Inhomogeneous self-affine carpets. - Indiana Univ. Math. J. 65, 2016, 15471566.

[12] Fraser, J. M., and T. Kempton: On the $L^{q}$ dimensions of measures on Heuter-Lalley type self-affine sets. - Proc. Amer. Math. Soc. 146, 2018, 161-173.

[13] Hata, M.: On some properties of set-dynamical systems. - Proc. Japan Acad. Ser. A 61, 1985, 99-102.

[14] Hueter, I., and S. P. LALley: Falconer's formula for the Hausdorff dimension of a self-affine set in $\mathbf{R}^{2}$. - Ergodic Theory Dynam. Systems 15:1, 1995, 77-97.

[15] Jordan, T., and N. JuRga: Self affine sets with non-compactly supported random perturbations. - Ann. Acad. Sci. Fenn. Math. 39, 2014, 771-785.

[16] KäEnmÄKI, A., and J. LEHRBÄCK: Measures with predetermined regularity and inhomogeneous self-similar sets. - Ark. Mat. 116:4, 2018, 929-956.

[17] Olsen, L., and N. Snigireva: $L^{q}$ spectra and Rényi dimensions of in-homogeneous self-similar measures. - Nonlinearity 20, 2007, 151-175.

[18] Snigireva, N.: Inhomogeneous self-similar sets and measures. - Ph.D. Dissertation, University of St Andrews, 2008.

Received 23 January 2019 • Accepted 24 May 2019 